\title{
Comparison of artifact generation with catheter bending using different PS-OCT approaches
}

\author{
This article was published in the following Dove Press journal: \\ Reports in Medical Imaging \\ 8 May 2009 \\ Number of times this article has been viewed
}

\author{
Kathy Zheng' \\ Christopher Rashidifard' \\ Bin Liu ${ }^{1,2}$ \\ Mark Brezinski ${ }^{1,2}$ \\ 'Center for Optical Coherence \\ Tomography and Optical Physics, \\ Department of Orthopedic Surgery, \\ Brigham and Women's Hospital, \\ Boston, MA, USA; ${ }^{2}$ Harvard Medical \\ School, Boston, MA, USA
}

\begin{abstract}
Optical coherence tomography (OCT) is a promising biomedical imaging tool for detecting pathology at a micron scale. In addition to performing structural imaging, other adjuvant techniques are available with OCT. In particular, polarization-sensitive optical coherence tomography (PS-OCT) can be used, either through a single or dual detector approach, to assess collagen concentration and organization. In this paper, we compare the ability of single and dual detector PS-OCT with respect to catheter bending artifacts. It was found that even severe catheter bending resulted in no significant difference in quantitative birefringence measurement with the single detector approach. However, when the dual detector approach was examined, catheter bending resulted in a highly significant change in backreflection intensity. This paper raises questions as to the appropriateness of dual detector PS-OCT for performing catheter and endoscope based birefringence assessments.
\end{abstract}

Keywords: Optical coherence tomography, polarization, single detector, endoscopic imaging

\section{Introduction}

Optical coherence tomography (OCT) is a promising biomedical imaging tool for detecting pathology at a micron scale. It has shown considerable potential as a diagnostic technology for a wide range of clinical scenarios. Its micron scale resolution is up to 100 times greater than anything available in clinical medicine..$^{1-3}$ Among its additional advantages are its high data acquisition rates, which now exceed 30 frames per second. In addition, OCT's fiber optics-based technology makes the system compact and portable, and most importantly integratable into extremely small catheters and endoscopes. Therefore, it represents a powerful technology for producing video rate assessments near the level of resolution of conventional biopsy.

In addition to performing high-resolution structural imaging, OCT has adjuvant capabilities. Among the most important of these is the assessment of tissue birefringence. Clinically relevant birefringent structures include enamel, dentin, nervous tissue, actin myosin complexes, and specifically collagen. ${ }^{4-10}$ Most importantly, polarization-sensitive optical coherence tomography (PS-OCT) has demonstrated the ability to assess collagen organization and concentration. Among the disorders where collagen disorganization represents a sign of early pathology are myocardial infarction and osteoarthritis. ${ }^{8,9}$ Previous work has shown that in tissues such as cartilage, atherosclerotic arteries, and tendon, where organized collagen is highly birefringent, the loss of polarization sensitivity detected by OCT results from the breakdown of the collagen architecture..$^{6,11-13}$
Correspondence: Mark Brezinsk Brigham and Women's Hospital, 75 Francis Street, MRBI I4, Boston, MA 02II5, USA

$\mathrm{Tel}+\mathrm{I} 6177326911$

Fax +l 6175258356

Email mebrezin@mit.edu 
PS-OCT can be performed through either dual detector or single detector approaches. In the dual detector approach (DDPS-OCT), the OCT system measures an absolute value of polarization at the detector, which is typically represented by either a Stokes or Jones vector. ${ }^{1,14-16}$ Later progress in this approach involves complicated polarization control/modulation and measurement for retrieving the birefringence information of the tissue in a fiber-based dual-detector system. ${ }^{17,18}$ Furthermore, by measuring the polarization state of the reflection from tissue surface in real time, the full birefringence data of the tissue can be retrieved with, to some degree, the artifact-reduction induced by catheter. ${ }^{19,20}$ While at first, measuring the absolute returning polarization state seems like the optimal approach, this mode of imaging is problematic for several reasons. First, the polarization state at the detector in most cases does not represent the backreflected polarization state from the tissue. This is because changes within the system, such as movement of fibers or temperature, alter the polarization of the backreflected signal. As a result, the true polarization state must be extrapolated through relatively complex and rarely completely effective means. In addition, it is the Muller's matrix that truly represents the tissue polarization altering capacity. This is significant because for each OCT image, as many as 16 birefringent images need to be analyzed to generate a Muller's matrix of tissue in one B-scan. This means the process is extremely time-consuming, complex, and not performed in real time. Also, the matrix generated is typically difficult to interpret by clinicians, making its value of question. This could be particularly problematic when the lone detector receiving signal also has a relatively low intensity. Another problem is that multiple filters and beam splitters are required, as seen in Figure 1, which lead to power loss and hinder real-time imaging.

Lastly, and most relevant to this paper, artifacts due to catheter bending or compression alter the birefringence of returning light, making correlation between tissue backreflected light and that at the detector even more difficult. Therefore, simply pulling back or bending the catheter can lead to fluctuations in the image, although later developments for this approach suppresses the motion artifacts to some degree. ${ }^{19,20}$

In contrast, single detector PS-OCT (SDPS-OCT) measures the change (relative) in the polarization either as a function of depth in the tissue or by changing the incident polarization state of the light. ${ }^{11-13,21}$ As shown in previous studies, tissue birefringence can be measured directly from the image in real time which has been confirmed in vitro.

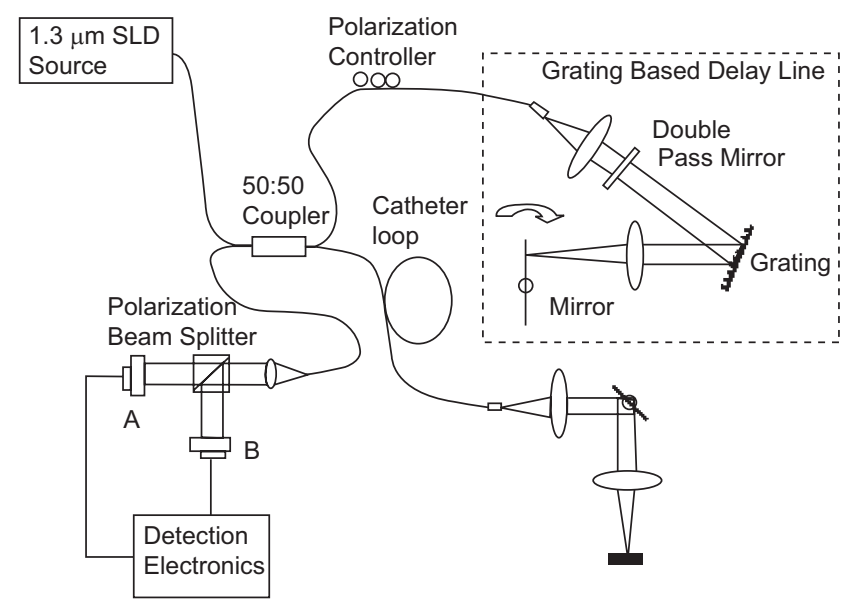

Figure I A schematic of the dual detector PS-OCT system used. The use of systems consisting of detectors $A$ and $B$ allow for measurements of orthogonal states. Abbreviation: PS-OCT, polarization-sensitive optical coherence tomography.

Second, birefringence can be measured as a function of depth. Third, for tissue with relatively low birefringence, the polarization state in the reference arm is rotated at constant velocity and time from intensity peak to the next is measured as shown. As a result of peak to peak measurements (not using trough data), noise and detector sensitivity play a minimal role. Finally, since only relative changes are measured in SDPS-OCT, ${ }^{22}$ artifacts from catheter bending or compression are essentially eliminated, which will be experimentally confirmed in this manuscript. Both SDPS-OCT and DDPS-OCT uses the same wideband light source, whose spectrum bandwidth determines the axial resolution of an OCT system. They have an identical resolution. Further interests in this regard can be found elsewhere..$^{23-25}$

The hypothesis of this paper is that polarization artifacts secondary to fiber bending are negligible with SDPS-OCT as compared with DDPS-OCT. This is particularly relevant in view of all the large number of catheter, endoscope, and other probe based OCT techniques currently being applied to clinical medicine.

\section{Methods}

Comparisons between single and dual detector PS-OCT was achieved using highly birefringent bovine Achilles' tendon using a LightLab ${ }^{\mathrm{TM}}$ OCT system (Westford, MA) The system is a time domain OCT with a median wavelength of $1287 \mathrm{~nm}$, an axial resolution of $12 \mu \mathrm{m}$, total power of $13 \mathrm{~mW}$, and an A-scan rate of $3.13 \mathrm{kHz}$. The system captures images at a rate of 10 frames per second $(800 \times 304$, radial $\times$ azimuthal $)$. The lateral resolution was $25 \mu \mathrm{m}$. Images of the same location on the tendon were taken in two different modes: SDPS-OCT or DDPS-OCT which the LightLab ${ }^{\mathrm{TM}}$ system 
has the capabilities of doing both. The schematic for dual detector imaging is shown in Figure 1. The dual detector imaging was performed such that birefringence is represented by blue. The birefringence is calculated as Eq. (1).

$$
\mathrm{I}_{\mathrm{B}}=\mathrm{C}[\log (\mathrm{A} / 256+1)-\log (\mathrm{B} / 256+1)] \approx \mathrm{Clog}(\mathrm{A} / \mathrm{B})
$$

$\mathrm{C}$ is a constant and $\mathrm{A}$ and $\mathrm{B}$ are the intensity values (16-bit numbers) from the orthogonal polarization channels $\mathrm{A}$ and $B$. The color is mapped into a red-blue color scale. The item $\mathrm{A} / \mathrm{B}$ is actually the first-order power series of the birefringence equation, ${ }^{26}$ where the phase retardance is given as $\arctan (\mathrm{A} / \mathrm{B})$. This approximation presents a simplified and rapid measurement with adequate accuracy. The logarithmic operation is for the display purpose.

For SDPS-OCT imaging shown in Figure 2, images were obtained with and without the polarization state being rotated through 180 degrees with polarization controllers in the reference arm as previously described. ${ }^{11-13}$

For meniscal tendon where the polarization state rotates rapidly within the tissue resulting in the banding pattern, there is no need to alter polarization states in the reference $\mathrm{arm}$. The process of rotating polarization in the reference arm for low birefringence tissue has been described extensively with our previous work. The change in backscattering as the paddles are rotated or as a function of depth (as in the case with the meniscus) is a measure of birefringence.

Images of the effects of catheter bending were taken in single and dual detector mode. The OCT imaging wire with a cross sectional diameter of $0.019^{\prime \prime}$ was placed $2 \mathrm{~mm}$ away at the focal point of the imaging beam from the surface of the tendon. First, the catheter was kept straight while imaging.

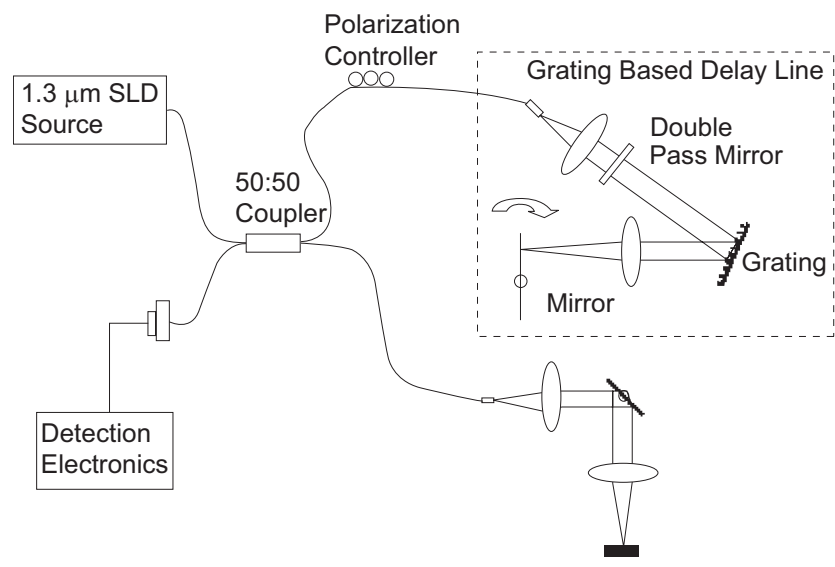

Figure 2 A schematic of the single detector PS-OCT system used. In this OCT system a polarization controller is placed in the reference arm, the advantages of which are described in the text.

Abbreviation: PS-OCT, polarization-sensitive optical coherence tomography.
Next, to induce constant angled catheter bending, the imaging wire was inserted through a plastic sheath $(\sim 15$ degrees/in $)$ to introduce multiple loops in the fiber, as seen in Figure 3.

The same sheath was used for all measurements. A visible light guiding beam was used, as the infrared OCT beam is not visible to the naked eye, which could be seen on the tissue and the surface marked with microinjections of dye to correlate with images. Video streams of the sample were saved in either AVI or MPEG format. Still frames from the videos were obtained during post-processing and saved in JPEG format.

For SDPS-OCT, the distance from peak to peak of each band was measured from six different still frames isolated from each video stream. The distances between peaks from images taken with a straight catheter and images taken with an extensively bent catheter were compared using Student's paired $t$-test. For all dual detector images, areas of about 120 pixels $\times 140$ pixels were isolated from random areas in the video stream. The same area in each image was analyzed with and without catheter bending. Intensity (per pixel) data of the region of interest from the images when the catheter was bent and the catheter straight was compared using Student's paired $t$-test and percent change was calculated.

After imaging, the Achilles' tendon samples were fixed in formalin for approximately 24 hours at room temperature followed by $0.1 \mathrm{M}$ phosphate-buffered saline. The tendon samples were processed for routine paraffin embedded sectioning. Picrosirius staining was used to visualize collagen concentration and organization. Its color, generated in part through an interference phenomenon, correlates with collagen type and fiber size. Large fibrils (greater than 60nm) are typically yellow to red and are generally collagen type I while fibrils in the range of 20-40 $\mathrm{nm}$ appear green and correlate with collagen type III. ${ }^{27,}{ }^{28}$ A high-resolution digital microscopy system with an Olympus BX-41 Microscope with a Q Color

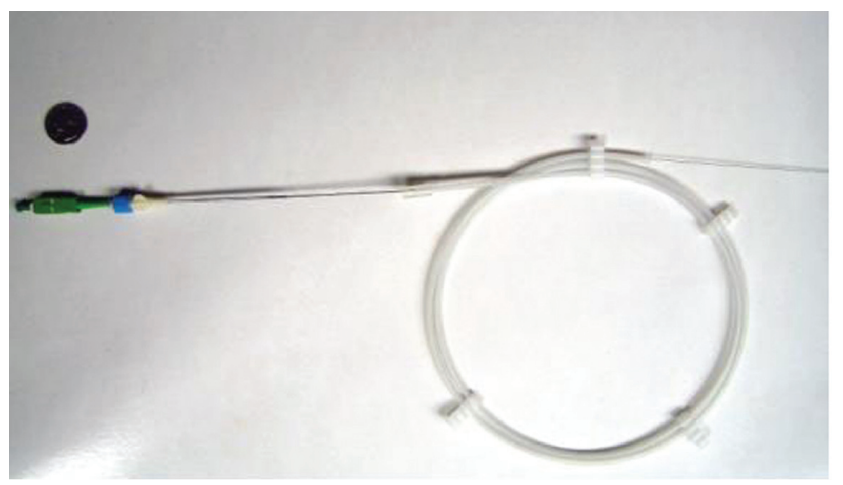

Figure 3 To induce constant angled catheter bending, the imaging wire is inserted through a plastic sheath to introduce multiple loops in the fiber. The same sheath was used for all measurements. 
3 CCD camera (Olympus America, Melville, NY) was used to produce digital photographs of the samples. An Olympus polarization filter (model UPOT) was used. The filter position was adjusted to the point where the background demonstrated no luminosity and the position was confirmed by the absence of colorization band artifacts. The capture software was Q Capture (Q Imaging Corp., Burnaby, Canada). The histology was evaluated for the presence of organized collagen.

\section{Results}

Bovine tendon was chosen because of the high concentration of well-organized collagen. The picrosirius histology section as seen in Figure 4 confirms the presence of highly organized collagen type I by the brightness in yellow color.

Images from the comparison between bent and straight catheter using SDPS-OCT are seen in Figure 5.

Pairwise Student's $t$-test comparison of mean banding distance between bent vs straight catheter showed no significant change in single detector mode $(n=6)$, as seen in Table 1 . A mean peak to peak distance of $50 \pm 5 \mu \mathrm{m}$ was measured when the catheter was bent and a mean distance of $54 \pm 8 \mu \mathrm{m}$ was measured when the catheter was straight.

Images from the comparison between bent and straight catheter using DDPS-OCT are seen in Figure 6.

In the dual detector mode as seen in Table 2, the backflection intensity changes in a given portion of tissue as the catheter is bent. In a paired $t$-test of the relative difference between mean intensities of dual detector images taken when the catheter is bent and when the catheter is straight, a highly statistically significant distance is noted $(\mathrm{p}<0.0001)$. The percent change of mean intensity for when the catheter is bent and when the catheter is straight was $29.34 \%$. This difference in intensities shows that the bending of the catheter could indeed lead to the erroneous interpretation of birefringence data with the dual detector approach.

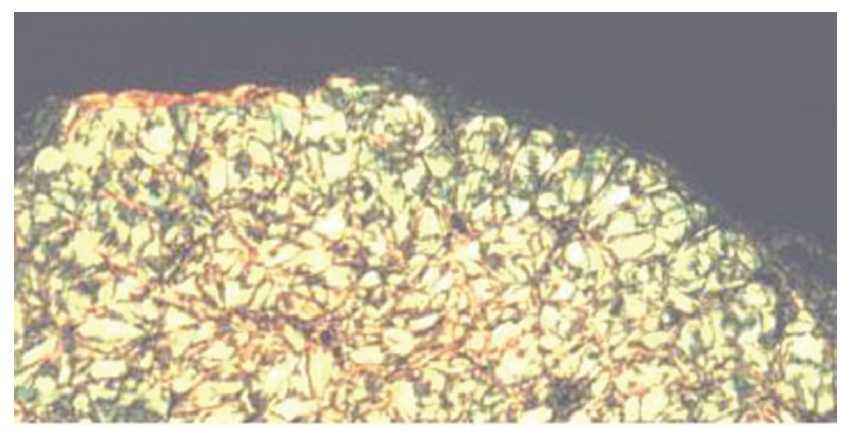

Figure 4 Bovine tendon was chosen because of the high concentration of well organized collagen. This picrosirius histology section confirms the presence of highly organized collagen type I by the brightness in yellow color.

\section{Bovine tendon - Single detector}

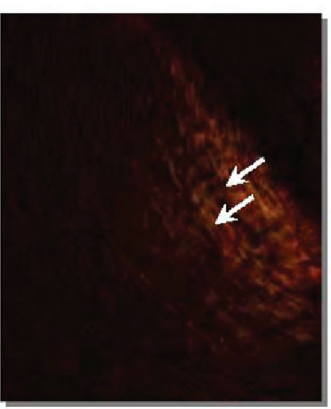

Bent catheter

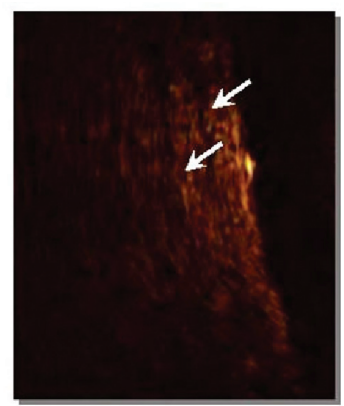

Straight catheter
Figure 5 Images from the comparison between bent and straight catheter using single detector PS-OCT. The technique utilizes relative rather than absolute birefringence to overcome many of the limitations of the dual detector approach. In this image of highly birefringent tissue, the banding pattern exists where bending of the catheter does not significantly alter distance between the bands and bending of the catheter. Pairwise Student's $t$-test comparison of mean banding distance between bent vs straight catheter showed no significant change although a slight shifting of the banding pattern (ie, no change in measured birefringence) can be seen. The peak to peak measurements also avoid the low signal and detector sensitivity issues found with DDPS-OCT.

Abbreviation: DDPS-OCT, dual detector polarization-sensitive optical coherence tomography.

\section{Discussion}

PS-OCT has significant clinical implications because, for example, of its ability to detect the breakdown of collagen architecture in highly birefringent tissue. We have demonstrated through previous work that the detection and analysis of collagen breakdown through PS-OCT is an important biomarker for the sensitive detection of diseases that take place within these birefringent tissues, producing a method for monitoring future therapeutic choices. ${ }^{11-13}$ OCT imaging can be performed through imaging catheters less than $0.019^{\prime \prime}$ in diameter. This is relevant for imaging procedures that monitor conditions such as atherosclerotic plaque progression or osteoarthritis development within a tight joint space. As a result, polarization artifacts that result from catheter bending

Table I Changes in the distance between bands with changes in the catheter bending in single detector mode. No significant difference was noted between straight and bent catheters

\begin{tabular}{llll}
\hline Sample & $\begin{array}{l}\text { Bent } \\
\text { (microns) }\end{array}$ & $\begin{array}{l}\text { Straight } \\
\text { (microns) }\end{array}$ & $\begin{array}{l}\text { Paired } \\
\text { t-test }\end{array}$ \\
\hline 1 & 51.351 & 50.512 & $P=0.07$ \\
2 & 51.737 & 62.116 & \\
3 & 54.826 & 55.290 & \\
4 & 50.965 & 58.020 & \\
5 & 50.965 & 52.560 & \\
6 & 40.154 & 50.512 & \\
Mean & $50 \pm 5$ & $54 \pm 8$ & \\
\hline
\end{tabular}




\section{Bovine tendon - Dual detector}

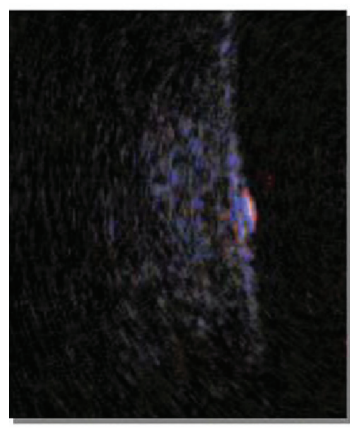

Straight catheter

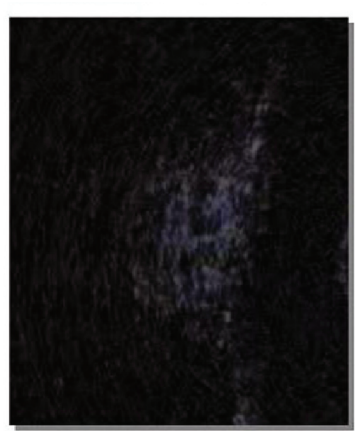

Bent catheter

Figure 6 Images from comparison between bent and straight catheter using dual detector PS-OCT. Birefringence is measured of the intensity and phase of the two orthogonal states entering the detector (and assumed to be representative of the tissue). When the catheter is bent, the incident light on the tissue changes its polarization state and the birefringence of light traveling in both directions is altered. It is noted in this image that a change in measured phase occurred as exemplified by the reduced intensity even though no actual change in tissue birefringence occurred. This difference in intensities shows that the bending of the catheter could lead to the erroneous interpretation of birefringence data with the dual detector approach In a paired $t$-test of the relative difference between mean intensities of dual detector images taken when the catheter is bent and when the catheter is straight, a significan difference was noted $(p<0.000 \mathrm{I})$.

Abbreviation: PS-OCT, polarization-sensitive optical coherence tomography.

or compression are especially significant for determining the validity of PS-OCT images.

In this work, we demonstrate the advantage of SDPS-OCT over DDPS-OCT. In single detector mode, birefringence is measured relative in the image rather than from an absolute value and quantified by the distance between peaks or bands. In highly birefringent tissue, such as with the Achilles' tendon used, the banding is seen with SDPS-OCT. The bending and unbending of the catheter used during imaging resulted in no significant change in the distance between bands produced as a result of the tissue birefringence (ie, relative distance between the bands remained constant).

Table 2 Changes in the backreflection intensity with and without catheter bending with DDPS-OCT. A highly significant difference was noted between straight and bent catheter conditions

\begin{tabular}{llll}
\hline Sample & $\begin{array}{l}\text { Bent (mean } \\
\text { intensity) }\end{array}$ & $\begin{array}{l}\text { Straight (mean } \\
\text { intensity) }\end{array}$ & Paired t-test \\
\hline 1 & 21.45 & 28.58 & $\mathrm{P}<0.0001$ \\
2 & 21.50 & 27.92 & \\
3 & 21.68 & 28.07 & \\
4 & 20.91 & 26.25 & \\
5 & 21.55 & 27.50 & \\
6 & 21.65 & 28.21 & \\
$\%$
\end{tabular}

Abbreviation: DDPS-OCT, dual detector polarization-sensitive optical coherence tomography.
This allows for constant birefringence measurements. The peak to peak measurements also avoid the low signal and detector sensitivity issues found with DDPS-OCT.

In the dual detector approach, birefringence is measured of the intensity and phase of the two orthogonal states entering the detector. When the catheter is bent, the incident light on the tissue changes its polarization state and the birefringence of light traveling in both directions is altered. As seen with the difference in intensities in the images in Figure 6, the bending of the catheter could lead to the erroneous interpretation of birefringence data with the dual detector approach. Also, it is noted that a change in measured phase occurred as exemplified by the reduced intensity even though no actual change in tissue birefringence occurred. In a paired t-test of the relative difference between mean intensities of dual detector images taken when the catheter is bent and when the catheter is straight, $\mathrm{p}<0.0001$. This raises the issue of how PS-OCT imaging can be performed through catheters and endoscopes using a dual detector approach. While some compensatory techniques have been applied, they are far from complete and often result in a reduction of acquisition rate. ${ }^{29,30}$

There were several limitations to this study. First, the results do not compare moderately or weakly birefringent tissue. This will be the subject of future work. Second, only one dual detector approach was examined but it was felt that this was the more viable for comparison (polarization diversity). Future studies may focus on comparisons of SDPS-OCT and DDPS-OCT within a variety of different tissue types.

In conclusion, this study demonstrates a superior ability of SDPS-OCT for assessing tissue birefringence via catheter based imaging compared with DDPS-OCT. This raises the important question as to how DDPS-OCT can be easily applied to minimally invasive and invasive OCT imaging.

\section{Acknowledgments}

This research is sponsored by the National Institutes of Health, contracts R01 AR44812, R01 HL55686, R01 EB02638/HL63953, R01 AR46996, and R01 EB000419. The authors would also like to thank Namita Kumar and Julie Williams for their technical support. The authors report no conflicts of interest in this work.

\section{References}

1. Brezinski ME. Optical Coherence Tomography: Principle and practice. Burlington, MA: Academic Press; 2006.

2. Huang D, Swanson EA, Lin CP, Schuman JS, Stinson WG, Chang W, et al Optical Coherence Tomography. Science. 1991;254(5035):1178-1181.

3. Brezinski ME, Tearney GJ, Bouma BE, Izatt JA, Hee MR, Swanson EA, et al. Optical coherence tomography for optical biopsy - Properties and demonstration of vascular pathology. Circulation. 1996;93(6):1206-1213. 
4. Herrmann JM, Pitris C, Bouma BE, et al. High resolution imaging of normal and osteoarthritic cartilage with optical coherence tomography. J Rheumatol. 1999;26(3):627-635.

5. Baumgartner A, Dichtl S, Hitzenberger CK, et al. Polarization-sensitive optical coherence tomography of dental structures. Caries Res. 2000; 34(1):59-69.

6. Martin SD, Patel NA, Adams SB, et al. New technology for assessing microstructural components of tendons and ligaments. Int Orthop. 2003;27(3):184-189.

7. Pasquesi JJ, Schlachter SC, Boppart MD, Chaney E, Kaufman SJ, Boppart SA. In vivo detection of exercise-induced ultrastructural changes in genetically-altered murine skeletal muscle using polarizationsensitive optical coherence tomography. Opt Express. 2006;14(4): 1547-1556.

8. Pierce MC, Sheridan RL, Park BH, Cense B, de Boer JF. Collagen denaturation can be quantified in burned human skin using polarizationsensitive optical coherence tomography. Burns. 2004;30(6):511-517.

9. Pierce MC, Strasswimmer J, Park BH, Cense B, de Boer JF. Birefringence measurements in human skin using polarization-sensitive optical coherence tomography. J Biomed Opt. 2004;9(2):287-291.

10. Strasswimmer J, Pierce MC, Park BH, Neel V, de Boer JF. Polarizationsensitive optical coherence tomography of invasive basal cell carcinoma. J Biomed Opt. 2004;9(2):292-298.

11. Drexler W, Stamper D, Jesser C, et al. Correlation of collagen organization with polarization sensitive imaging of in vitro cartilage: Implications for osteoarthritis. J Rheumatol. 2001;28(6):1311-1318.

12. Giattina SD, Courtney BK, Herz PR, Harman M, Shortkroff S, Stamper DL, et al. Assessment of coronary plaque collagen with polarization sensitive optical coherence tomography (PS-OCT). Int J Cardiol. 2006;107(3):400-409.

13. Li XD, Martin S, Pitris C, et al. High-resolution optical coherence tomographic imaging of osteoarthritic cartilage during open knee surgery. Arthritis Res Ther. 2005;7(2):R318-R323.

14. Jiao SL, Wang LHV. Jones-matrix imaging of biological tissues with quadruple-channel optical coherence tomography. J Biomed Opt. 2002;7(3):350-358.

15. Jiao SL, Wang LHV. Two-dimensional depth-resolved Mueller matrix of biological tissue measured with double-beam polarization-sensitive optical coherence tomography. Opt Lett. 2002;27(2):101-103.

16. de Boer JF, Milner TE. Review of polarization sensitive optical coherence tomography and Stokes vector determination. J Biomed Opt. 2002;7(3):359-371.

17. Park BH, Pierce MC, Cense B, de Boer JF. Real-time multi-functional optical coherence tomography. Opt Express. 2003;11(7):782-793.
18. Saxer CE, de Boer JF, Park BH, Zhao YH, Chen ZP, Nelson JS. High-speed fiber-based polarization-sensitive optical coherence tomography of in vivo human skin. Opt Lett. 2000;25(18):1355-1357.

19. Oh WY, Yun SH, Vakoc BJ, Shishkov M, Desjardins AE, Park BH, et al. High-speed polarization sensitive optical frequency domain imaging with frequency multiplexing. Opt Express. 2008;16(2):1096-1103.

20. Pierce MC, Shishkov M, Park BH, et al. Effects of sample arm motion in endoscopic polarization-sensitive optical coherence tomography. Opt Express. 2005;13(15):5739-5749.

21. Liu B, Harman M, Giattina S, et al. Characterizing of tissue microstructure with single-detector polarization-sensitive optical coherence tomography. Appl Opt. 2006;45(18):4464-4479.

22. Liu B, Harman M, Brezinski ME. Variables affecting polarizationsensitive optical coherence tomography imaging examined through the modeling of birefringent phantoms. J Opt Soc Am A Opt Image Sci Vis. 2005;22(2):262-271.

23. Bagnaninchi PO, Churmakov D, Bonesi M, et al. Optical properties of human tendons characterized by PSOCT and their relation to tendinopathy: a clinical study. Optical Interactions with Tissue and Cells XIX. 2008;6854:68541C.

24. Ugryumova N, Attenburrow DP, Winlove CP, Matcher SJ. The collagen structure of equine articular cartilage, characterized using polarization-sensitive optical coherence tomography. J Phys D Appl Phys. 2005;38(15):2612-2619.

25. Matcher SJ, Winlove CP, Gangnus SV. The collagen structure of bovine intervertebral disc studied using polarization-sensitive optical coherence tomography. Phys Med Biol. 2004;49(7):1295-1306.

26. Schoenenberger K, Colston BW, Maitland DJ, Da Silva LB, Everett MJ. Mapping of birefringence and thermal damage in tissue by use of polarization-sensitive optical coherence tomography. Appl Opt. 1998;37(25):6026-6036.

27. Junqueira LCU, Figueiredo MTA, Torloni H, Montes GS. Differential histologic diagnosis of osteoid - a study on human osteosarcoma collagen by the histochemical picrosirius-polarization method. J Pathol. 1986;148(2):189-\&.

28. Junqueira LCU, Bignolas G, Brentani RR. Picrosirius staining plus polarization microscopy, a specific method for collagen detection in tissue-sections. Histochem J. 1979;11(4):447-455.

29. Jiao SL, Yu WR, Stoica G, Wang LHV. Optical-fiber-based Mueller optical coherence tomography. Opt Lett. 2003;28(14):1206-1208.

30. Park BH, Pierce MC, Cense B, de Boer JF. Jones matrix analysis for a polarization-sensitive optical coherence tomography system using fiber-optic components. Opt Lett. 2004;29(21):2512-2514.
Reports in Medical Imaging

\section{Publish your work in this journal}

Reports in Medical Imaging is an international, peer-reviewed, open access journal publishing original research, reports, reviews and commentaries on all areas of medical imaging. The manuscript management system is completely online and includes a very quick and fair peer-review system, which is all easy to use.

\section{Dovepress}

Visit http://www.dovepress.com/testimonials.php to read real quotes from published authors. 